\title{
Hamiltonian formulation of the secular theory for Trojan-type motion
}

\author{
M. H. M. Morais` \\ Observatoire de la Côte d'Azur, BP 4229, 06304 Nice Cedex 4, France \\ Received 19 October 2000 / Accepted 12 January 2001

\begin{abstract}
We re-derive the secular theory for Trojan-type motion from Morais (1999) using a Hamiltonian formulation and show how this methodology allows us to include the effect of an oblate central mass and the secular perturbations from additional bodies in a rigorous way. As an application of this work we locate secular resonances inside the co-orbital regions of the uranian satellites and the planets, and show that these are in good agreement with the behaviour observed in numerical integrations.
\end{abstract}

Key words. celestial mechanics - minor planets, asteroids - planets and satellites: general

\section{Introduction}

Lagrange (1867-1892) showed that the three-body problem has five relative equilibrium configurations. In the restricted version (i.e. when one of the bodies is massless) it is useful to view the motion of the test particle in a frame co-rotating with the massive bodies; in this case the relative equilibrium configurations correspond to fixed points two of which, named $L_{4}$ and $L_{5}$, are linearly stable when the mass ratio is small enough (i.e. when $\left.m_{1} / m_{\mathrm{c}}<0.0385\right)$. These are also known as the triangular equilibrium points and correspond to configurations where the test particle is in the exact 1:1 mean motion resonance, i.e. it is co-orbital ${ }^{1}$ with $m_{1}$ but leading or trailing it by $60^{\circ}$. In this paper, Trojan-type motion refers to the tadpole-shaped librations around $L_{4}$ or $L_{5}$ (in analogy with the Trojan asteroids which occupy tadpole orbits in the Sun-Jupiter system) and also to the horseshoe-shaped librations that enclose both triangular points as well as the unstable co-linear point $L_{3}$.

In Morais (1999), hereafter Paper I, we constructed a secular theory for Trojan-type motion in the framework of the restricted three-body problem. A secular solution was derived based on the heuristic assumption that the terms in the averaged disturbing potential depending on the relative mean longitude do not have a net effect on the evolution of the eccentricity or inclination. Here we

\footnotetext{
* e-mail: morais@obs-nice.fr

1 The word co-orbital, which has here its literal meaning of shares the orbit with, is also generally used whenever referring to any nearby orbits.
}

show how we can re-derive this secular solution applying first-order canonical adiabatic theory (Lichtenberg \& Lieberman 1983). Moreover, we show that this methodology allows us to construct a complete secular theory which includes the effect of an oblate central mass and the secular perturbations from additional massive bodies, and we are thus able to explain rigorously some preliminary results obtained in Paper I.

\section{Settings of the perturbation scheme and the short-term behaviour}

\subsection{Restricted three-body case}

Consider the hierarchical restricted three-body problem with $m_{\mathrm{c}} \gg m_{1}$. The equation that describes the motion in the vicinity of $L_{4}$ or $L_{5}$ is

$\ddot{\boldsymbol{r}}=-\mathcal{G} \frac{\left(m_{\mathrm{c}}+m_{1}\right)}{r^{3}} \boldsymbol{r}+\nabla \mathcal{R}$

where the disturbing potential $\mathcal{R}$ is defined as

$\mathcal{R}=\mathcal{G} m_{1}\left(\frac{1}{\left|\boldsymbol{r}_{1}-\boldsymbol{r}\right|}-\frac{\boldsymbol{r} \cdot \boldsymbol{r}_{1}}{r_{1}^{3}}-\frac{1}{r}\right)$

with $\boldsymbol{r}$ and $\boldsymbol{r}_{1}$ being, respectively, the vector positions of the test particle and mass $m_{1}$ relative to the mass $m_{\mathrm{c}}$.

Note that the second term on the right hand side of Eq. (1) vanishes at the triangular points; this means that a test particle located at $L_{4}$ (or $L_{5}$ ) describes a keplerian ellipse which has an equal size, equal shape and common focus to that described by the mass $m_{1}$, but whose orientation is shifted by $60^{\circ}$ ( or $-60^{\circ}$ ) with respect to the latter. 
As usual we define the set $(a, e, I, \lambda, \varpi, \Omega)$ as the osculating elements (respectively, semi-major axis, eccentricity, inclination, mean longitude, longitude of pericentre, longitude of node) of the test particle's orbit. Also, in our notation, unsubscribed quantities refer to the test particle and quantities with subscript $k$ refer to a mass $m_{k}$. We then define the average angular velocity around the two-body ellipse (or mean motion) as $n=\dot{\lambda}$, and we relate this to the semi-major axis $a$ by $^{2}$

$n^{2} a^{3}=\mathcal{G}\left(m_{\mathrm{c}}+m_{1}\right)$.

In Paper I we first expressed the disturbing potential $\mathcal{R}$ in terms of osculating elements including terms up to degree two in eccentricities and inclinations; then we performed a change of variables $\left(\lambda, \lambda_{1}\right) \rightarrow\left(\phi=\lambda-\lambda_{1}, \lambda_{1}\right)$ and averaged $\mathcal{R}$ with respect to the fast angle $\lambda_{1}$. The averaged expansion of the disturbing potential is

$$
\begin{aligned}
\mathcal{R}_{1: 1}= & n^{2} a^{2} \mu_{1} \frac{a}{a_{1}}\left(\left[\frac{a^{2}}{a_{1}^{2}}+1-2 \frac{a}{a_{1}} \cos \phi\right]^{-1 / 2}\right. \\
& \left.-\frac{a}{a_{1}} \cos \phi-\frac{a_{1}}{a}+X\right)
\end{aligned}
$$

with

$$
\begin{aligned}
X= & g_{1}(\phi) e^{2}+g_{2}(\phi) e e_{1} \cos \left(\varpi-\varpi_{1}\right) \\
& +g_{3}(\phi) e e_{1} \sin \left(\varpi-\varpi_{1}\right) \\
& +g_{4}(\phi) I^{2}+g_{5}(\phi) I I_{1} \cos \left(\Omega-\Omega_{1}\right) \\
& +g_{6}(\phi) I I_{1} \sin \left(\Omega-\Omega_{1}\right)
\end{aligned}
$$

where $\mu_{1}=m_{1} /\left(m_{\mathrm{c}}+m_{1}\right)$, and we refer to Paper I for the explicit form of the functions $g_{i}(\phi)$.

Tadpole and horseshoe orbits can be decomposed into a slow guiding centre motion described by the variables $\delta a=a-a_{1}$ and $\phi$, with a superimposed fast epicycle due to motion from pericentre to apocentre viewed in the frame co-rotating with $m_{1}$. Averaging over the mean longitude $\lambda_{1}$ effectively removes the epicyclic motion and is justified in terms of perturbation theory by the fact that the amplitude of the epicycle is an adiabatic invariant. This is not only confirmed by numerical experiments (e.g. Dermott \& Murray 1981) but has also been proved rigorously in the framework of Hill's problem (Henon \& Petit 1986). Note, however, that the adiabatic invariance breaks down if the test particle can get close to the mass $m_{1}$. Indeed, numerical experiments (Dermott \& Murray 1981; Gladman 1993) show that near-circular and near-planar orbits with $0.74 \epsilon<\delta a / a_{1}<3.5 \epsilon\left(\right.$ where $\left.\epsilon=\left(\mu_{1} / 3\right)^{1 / 3}\right)$ are strongly affected by successive encounters with $m_{1}$, thus being chaotic and potentially unstable. The adiabatic invariance also breaks down in the vicinity of the separatrix between the tadpole and horseshoe regions where there is overlap of high order resonances between the angles $\phi$ and $\lambda$.

\footnotetext{
${ }^{2}$ Note that Eq. (3) implies that at $L_{4}$ (or $L_{5}$ ), $a=a_{1}$ and $\lambda-\lambda_{1}=+60^{\circ}$ (or $-60^{\circ}$ ) and follows from the fact that the three-body potential at $L_{4}$ (or $L_{5}$ ) is of keplerian type but with a larger mean motion.
}

\subsubsection{The guiding centre's motion}

We now want to obtain the equation of the guiding centre (that gives the evolution of $\delta a$ with $\phi$ ). From Lagrange's planetary equations we have

$$
\begin{aligned}
\dot{\phi}= & n-n_{1}-\frac{2}{n a} \frac{\partial \mathcal{R}_{1: 1}}{\partial a}+\frac{\tan \frac{1}{2} I}{n a^{2} \sqrt{1-e^{2}}} \frac{\partial \mathcal{R}_{1: 1}}{\partial I} \\
& +\frac{\sqrt{1-e^{2}}\left(1-\sqrt{1-e^{2}}\right)}{n a^{2} e} \frac{\partial \mathcal{R}_{1: 1}}{\partial e} \\
\delta \dot{a}= & \frac{2}{n a} \frac{\partial \mathcal{R}_{1: 1}}{\partial \phi} .
\end{aligned}
$$

Following Paper I we note that $\delta a=\mathcal{O}\left(\sqrt{\mu_{1}} a_{1}\right)$; hence

$\dot{\phi}=-\frac{3}{2} \frac{\delta a}{a_{1}} n_{1}+\mathcal{O}\left(\mu_{1} n_{1}\right)$.

Moreover, if we assume that the eccentricities and the inclinations are small, i.e. at most $\mathcal{O}\left(\mu_{1}^{1 / 4}\right)$, then we have

$\frac{\delta \dot{a}}{a_{1}}=2 \mu_{1} n_{1} \frac{\mathrm{d} f(\phi)}{\mathrm{d} \phi}+\mathcal{O}\left(\mu_{1}^{3 / 2} n_{1}\right)$

where

$f(\phi)=\frac{1+4|\sin (\phi / 2)|^{3}}{2|\sin (\phi / 2)|}$.

From Eqs. (8) and (9), neglecting terms $\mathcal{O}\left(\mu_{1}^{3 / 2} n_{1}^{2}\right)$, we obtain

$\ddot{\phi}=-3 \mu_{1} n_{1}^{2} \frac{\mathrm{d} f(\phi)}{\mathrm{d} \phi}$.

This equation (which can also be found in the work of Yoder et al. 1983) has an integral of the motion

$\mu_{1} n_{1}^{2} E=-\frac{1}{6} \dot{\phi}^{2}-\mu_{1} n_{1}^{2} f(\phi)<0$.

Now from Eqs. (8) and (12) we obtain the equation of the guiding centre

$\frac{\delta a}{a_{1}}= \pm \sqrt{\frac{8}{3} \mu_{1}(-E-f(\phi))}$

and as $f(\phi)$ has minima at $\phi= \pm \pi / 3$, the maximum value of $|\delta a| / a_{1}$ occurs at $\phi= \pm \pi / 3$ and is given by

$a_{0}=\sqrt{\frac{8}{3} \mu_{1}\left(-E-\frac{3}{2}\right)}$.

In Fig. 1 we show some guiding centre trajectories described by Eq. (13). Orbits with $3 / 2<-E<5 / 2$ are tadpole-shaped and orbits with $-E>5 / 2$ are horseshoeshaped. The separatrix curve (i.e. the boundary between the tadpole and horseshoe regions) has $-E=5 / 2$.

Alternatively, we can describe the motion of the guiding centre using the integrable Hamiltonian

$H_{0}=-\frac{3}{8} n_{1}^{2} a_{1}^{2}\left(\frac{\delta a}{a_{1}}\right)^{2}-\mu_{1} n_{1}^{2} a_{1}^{2} f(\phi)=\mu_{1} n_{1}^{2} a_{1}^{2} E$ 


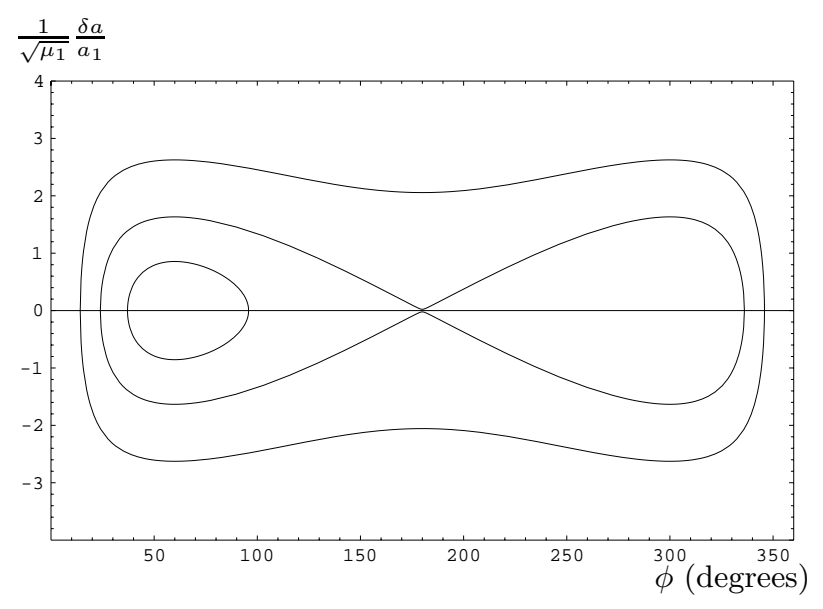

Fig. 1. Guiding centre trajectories obtained with Eq. (13): $-E=1.77465$ (inner curves), $-E=2.50019$ (separatrix) and $-E=4.08606$ (outer curve). Note that $f(\phi)$ is symmetric about $\phi=180^{\circ}$; in particular this implies that when $-E=1.77465$ there is another possible trajectory which is symmetric with respect to the one shown here

whose trajectories have periodicity

$T_{1: 1}=\oint \frac{\mathrm{d} \phi}{\dot{\phi}}$

and which admits the action-angle variables $(J, \theta)$, such that

$J=H_{0} \frac{T_{1: 1}}{2 \pi}$

$\dot{\theta}=\frac{2 \pi}{T_{1: 1}}$.

\subsubsection{Expansion of the Hamiltonian}

The time-independent Hamiltonian of the restricted threebody problem can be written as

$H=-\frac{\left(\mathcal{G}\left(m_{\mathrm{c}}+m_{1}\right)\right)^{2}}{2 \Lambda^{2}}-\mathcal{R}+\Lambda_{1} n_{1}$

where we used the following angle-action variables of the isolated two-body systems

$\lambda$

$$
\begin{aligned}
& \Lambda=\sqrt{\mathcal{G}\left(m_{\mathrm{c}}+m_{1}\right) a} \\
& \Lambda_{1}=\sqrt{\mathcal{G}\left(m_{\mathrm{c}}+m_{1}\right) a_{1}} .
\end{aligned}
$$

In order to obtain Eq. (4) we performed a canonical transformation to the following angle-action variables

$$
\begin{array}{ll}
\hat{\lambda}=\lambda-\lambda_{1} & \hat{\Lambda}=\Lambda \\
\hat{\lambda}_{1}=\lambda_{1} & \hat{\Lambda}_{1}=\Lambda_{1}+\Lambda .
\end{array}
$$

Therefore, the time-independent Hamiltonian of the averaged restricted three-body problem in the vicinity of the $1: 1$ mean motion resonance is

$\hat{H}=-\frac{\left(\mathcal{G}\left(m_{\mathrm{c}}+m_{1}\right)\right)^{2}}{2 \hat{\Lambda}^{2}}-\hat{\Lambda} n_{1}+\hat{\Lambda}_{1} n_{1}-\mathcal{R}_{1: 1}$ and as there is no dependence on $\hat{\lambda}_{1}$, the third term on the right hand side of Eq. (22) is constant and thus can be dropped.

It is straightforward to obtain again $H_{0}$ (given by Eq. (15)) by expanding Eq. (22) in powers of $\delta a / a_{1}$, assuming that the eccentricities and the inclinations are at most $\mathcal{O}\left(\mu_{1}^{1 / 4}\right)$, and keeping only the terms up to $\mathcal{O}\left(\mu_{1} n_{1}^{2} a_{1}^{2}\right)$ while dropping any constant terms. We can then expand the Hamiltonian of the averaged problem (Eq. (22)) to first-order in the small parameter $\sqrt{\mu_{1}}$, i.e. we write

$H=H_{0}+\sqrt{\mu_{1}} H_{1}$

where $H_{0}$ is given by Eq. (15) and

$\sqrt{\mu_{1}} H_{1}=n_{1}^{2} a_{1}^{2}\left(\frac{7}{16}\left(\frac{\delta a}{a_{1}}\right)^{3}+\mu_{1} f(\phi)\right)-\mathcal{R}_{1: 1}$.

It is important to recall here the assumption of small eccentricities and inclinations made in the derivation of the guiding centre's equation (Eq. (11)) and in the expansion of the Hamiltonian (Eq. (23)). As under this approximation the zero-order part of the Hamiltonian, $H_{0}$, is independent of $e$ or $I$, we obtained guiding centre trajectories which are stationary with respect to the secular perturbations. Therefore, we do not consider here the co-orbital structures whose guiding centres are modulated by the secular evolution, which have been studied by Namouni (1999) in the framework of the three-body problem.

\subsection{The effect of an oblate planet}

Now suppose that the body with mass $m_{\mathrm{c}}$ has an oblate shape with equatorial radius $R$ and that we can consider only the dominant zonal harmonic term (with coefficient $J_{2}$ ) in the oblateness disturbing potential. It can be shown (Kozai 1959) that the effect of the oblate planet on a satellite's orbit can be modeled by averaging the disturbing potential over the mean longitude, i.e. by taking into account only the secular part of the oblateness disturbing potential, which is

$\overline{\mathcal{R}}_{\text {obl }}=n a^{2} \beta(a)\left(\frac{1}{3}-\frac{1}{2} \sin ^{2} I\right)\left(1-e^{2}\right)^{-3 / 2}$

with

$\beta(a)=\frac{3}{2} J_{2}\left(\frac{R}{a}\right)^{2} \sqrt{\mathcal{G} m_{\mathrm{c}}} a^{-3 / 2}$.

The potential $\overline{\mathcal{R}}_{\text {obl }}$ induces the following precession rates in the satellite's orbit

$$
\begin{aligned}
\dot{\varpi} & =\frac{\sqrt{1-e^{2}}}{n a^{2} e} \frac{\partial \overline{\mathcal{R}}_{\mathrm{obl}}}{\partial e}+\frac{\tan \frac{1}{2} I}{n a^{2} \sqrt{1-e^{2}}} \frac{\partial \overline{\mathcal{R}}_{\mathrm{obl}}}{\partial I} \\
& =\beta(a)\left(1-e^{2}\right)^{-2}\left[1-\frac{3}{2} \sin ^{2} I-\tan \frac{I}{2} \sin I \cos I\right] \\
& \approx \beta(a) \\
\dot{\Omega} & =\frac{1}{n a^{2} \sqrt{1-e^{2}} \sin I} \frac{\partial \overline{\mathcal{R}}_{\mathrm{obl}}}{\partial I} \\
& =-\beta(a)\left(1-e^{2}\right)^{-2} \cos I \approx-\beta(a)
\end{aligned}
$$


and it also introduces the following correction to the mean motion of the satellite's orbit

$$
\begin{aligned}
\dot{\lambda}= & n-\frac{2}{n a} \frac{\partial \overline{\mathcal{R}}_{\mathrm{obl}}}{\partial a}+\frac{\tan \frac{1}{2} I}{n a^{2} \sqrt{1-e^{2}}} \frac{\partial \overline{\mathcal{R}}_{\mathrm{obl}}}{\partial I} \\
& +\frac{\sqrt{1-e^{2}}\left(1-\sqrt{1-e^{2}}\right)}{n a^{2} e} \frac{\partial \overline{\mathcal{R}}_{\mathrm{obl}}}{\partial e} \approx n+2 \beta(a) .
\end{aligned}
$$

We now want to see what the effect of an oblate planet is on the guiding centre's motion of satellites in tadpole or horseshoe orbits. As there are no perturbations on the semi-major $\operatorname{axis}^{3}$ we only have to take into account the contribution of the secular oblateness potential to $\dot{\phi}=$ $\dot{\lambda}-\dot{\lambda}_{1}$ which from Eq. (29) is $2 \beta(a)-2 \beta\left(a_{1}\right)$. Adding this to the right hand side of Eq. (8) yields

$$
\dot{\phi}=-\frac{3}{2} \frac{\delta a}{a_{1}} n_{1}\left(1+7 J_{2}\left(\frac{R}{a_{1}}\right)^{2}\right)+\mathcal{O}\left(\mu_{1} n_{1}\right) .
$$

Then, from Eqs. (30) and (9), we obtain an integral of the $\phi$-motion similar to Eq. (12). Therefore, the motion of the guiding centre is described by an integrable Hamiltonian $H_{0}$, which has the same form as Eq. (15). So again we write the Hamiltonian of the averaged problem as

$H=H_{0}+\sqrt{\mu_{1}} H_{1}$

where now

$\sqrt{\mu_{1}} H_{1}=n_{1}^{2} a_{1}^{2}\left(\frac{7}{16}\left(\frac{\delta a}{a_{1}}\right)^{3}+\mu_{1} f(\phi)\right)-\mathcal{R}_{1: 1}-\overline{\mathcal{R}}_{\mathrm{obl}}$.

As the orbit of the mass $m_{1}$ precesses due to the oblateness, the Hamiltonian given by Eq. (31) depends explicitly on time. If we assume that $\dot{\varpi}=-\dot{\Omega}=\beta(a)=\beta$ and $\dot{\varpi}_{1}=-\dot{\Omega}_{1}=\beta\left(a_{1}\right)=\beta_{1}$, one can avoid this explicit time dependence by introducing the following two extra degrees of freedom and associated conjugate momenta

$$
\begin{array}{ll}
\lambda_{\mathrm{g} 1}=-\beta_{1} t-\chi_{1} & \Lambda_{\mathrm{g} 1} \\
\lambda_{\mathrm{f} 1}=\beta_{1} t-\Xi_{1} & \Lambda_{\mathrm{f} 1}
\end{array}
$$

and by adding the terms $-\Lambda_{\mathrm{g} 1} \beta_{1}$ and $\Lambda_{\mathrm{f} 1} \beta_{1}$ to the new Hamiltonian.

\subsection{The effect of additional massive bodies}

Now consider $N$ massive bodies orbiting a primary of mass $m_{\mathrm{c}}$. It is well known (see e.g. Brouwer \& Clemence 1961) that in the absence of mean motion resonances between the $N$ massive bodies, one can obtain a good description of the long-term behaviour of, say $m_{j}$, by averaging the disturbing potential over the mean longitudes. The expansion of this secular disturbing potential, up to degree two

\footnotetext{
${ }^{3}$ Here we refer to a semi-major axis obeying the approximate relation $n^{2} a^{3}=\mathcal{G} m_{\mathrm{c}}\left(1+(3 / 2) J_{2}\left(R / a_{1}\right)^{2}\right)$ which follows from the fact that the oblateness potential is of nearly keplerian type but with a larger mean motion (see Greenberg 1981).
}

in eccentricities and inclinations, is

$$
\begin{aligned}
\overline{\mathcal{R}}_{j}= & n_{j} a_{j}^{2}\left(\frac{A_{j, j}}{2} e_{j}^{2}+\sum_{i \neq j} A_{j, i} e_{j} e_{i} \cos \left(\varpi_{j}-\varpi_{i}\right)\right. \\
& \left.+\frac{B_{j, j}}{2} I_{j}^{2}+\sum_{i \neq j} B_{j, i} I_{j} I_{i} \cos \left(\Omega_{j}-\Omega_{i}\right)\right)
\end{aligned}
$$

where the elements of the matrices $A$ and $B$ are functions of the masses, mean motions and semi-major axes of the massive bodies, whose definition can be found in Paper I.

The secular solution for the mass $m_{j}$ is simply

$$
\begin{aligned}
e_{j} \exp \left[\mathrm{i} \varpi_{j}\right] & =\sum_{i} e_{j, i} \exp \left[\mathrm{i}\left(g_{i} t+\chi_{i}\right)\right] \\
I_{j} \exp \left[\mathrm{i} \Omega_{j}\right] & =\sum_{i} I_{j, i} \exp \left[\mathrm{i}\left(f_{i} t+\Xi_{i}\right)\right]
\end{aligned}
$$

where the frequencies $g_{i}$ (and $f_{i}$ ) are the eigen-values of the matrix $A$ (and $B$ ), the coefficients $e_{j, i}$ (and $I_{j, i}$ ) are the components of the eigen-vectors associated with $g_{i}$ (and $f_{i}$ ), and the phases $\chi_{i}$ (and $\Xi_{i}$ ) are constants determined by the initial conditions.

Moreover, the secular disturbing potential acting on a test particle in a tadpole or horseshoe orbit associated with the mass $m_{k}$, due to the masses $m_{j}$ (where $j \neq k$ ), expanded up to degree two in eccentricities and inclinations, is

$$
\begin{aligned}
\overline{\mathcal{R}}_{0, k}= & n a^{2}\left(\frac{A_{k}}{2} e^{2}+\sum_{j \neq k} A_{j} e e_{j} \cos \left(\varpi-\varpi_{j}\right)\right. \\
& \left.+\frac{B_{k}}{2} I^{2}+\sum_{j \neq k} B_{j} I I_{j} \cos \left(\Omega-\Omega_{j}\right)\right)
\end{aligned}
$$

with $A_{i}$ (and $B_{i}$ ) defined as $A_{k, i}\left(\right.$ and $\left.B_{k, i}\right)$ but with $m_{k}=$ $0, a_{k}$ replaced by $a$, and $n_{k}$ replaced by $n$.

We now want to see how the secular perturbations from these additional massive bodies change the guiding centre's motion of tadpole or horseshoe orbits. Obviously there are no secular perturbations to the semi-major axis. Now, adding to the right hand side of Eq. (8) the contribution of the additional massive bodies to $\dot{\phi}=\dot{\lambda}-\dot{\lambda}_{k}$, yields

$\dot{\phi}=-\frac{3}{2} \frac{\delta a}{a_{k}} n_{k}\left(1+b \sum_{i<k} \frac{m_{i}}{m_{\mathrm{c}}}+\tilde{b} \sum_{i>k} \frac{m_{i}}{m_{\mathrm{c}}}\right)+\mathcal{O}\left(\mu_{k} n_{k}\right)$

where $b$ and $\tilde{b}$ are functions of the semi-major axes of the massive bodies.

Then, from Eqs. (38) and (9), we obtain an integral of the $\phi$-motion similar to Eq. (12). Therefore, the motion of the guiding centre is still described by an integrable Hamiltonian $H_{0}$, which has the same form as Eq. (15). So once more we write the Hamiltonian of the averaged problem as

$H=H_{0}+\sqrt{\mu_{k}} H_{1}$ 
where now

$\sqrt{\mu_{k}} H_{1}=n_{k}^{2} a_{k}^{2}\left(\frac{7}{16}\left(\frac{\delta a}{a_{k}}\right)^{3}+\mu_{k} f(\phi)\right)-\mathcal{R}_{1: 1}-\overline{\mathcal{R}}_{0, k}$.

Note that although this Hamiltonian depends explicitly on time through the relations in Eqs. (35) and (36), this can be avoided by over-extending the phase space to include the following extra $2 N$ degrees of freedom and associated conjugate momenta

$$
\begin{array}{ll}
\lambda_{\mathrm{g} i}=-g_{i} t-\chi_{i} & \Lambda_{\mathrm{g} i} \\
\lambda_{\mathrm{f} i}=-f_{i} t-\Xi_{i} & \Lambda_{\mathrm{f} i}
\end{array}
$$

and by adding the terms $-\sum_{i} \Lambda_{\mathrm{g} i} g_{i}$ and $-\sum_{i} \Lambda_{\mathrm{f} i} f_{i}$ to the new Hamiltonian.

\section{The long-term behaviour: Secular theory}

\subsection{Restricted three-body case}

We now recall Sect. 2.1.2 where we obtained the Hamiltonian of the averaged problem (Eq. (23)) and then apply Eq. (13) in order to write its first-order part (Eq. (24)) as

$\sqrt{\mu_{1}} H_{1}=\mu_{1} n_{1}^{2} a_{1}^{2} F(\phi) \frac{\delta a}{a_{1}}-X$

where $F(\phi)$ is a function of $\phi$,

$$
\begin{aligned}
X= & \mu_{1} n_{1}\left[g_{1}(\phi) 2 M+g_{4}(\phi) 2 N\right. \\
& +g_{2}(\phi) \sqrt{n} a(2 M)^{1 / 2} e_{1} \cos \left(\varpi-\varpi_{1}\right) \\
& +g_{3}(\phi) \sqrt{n} a(2 M)^{1 / 2} e_{1} \sin \left(\varpi-\varpi_{1}\right) \\
& +g_{5}(\phi) \sqrt{n} a(2 N)^{1 / 2} I_{1} \cos \left(\Omega-\Omega_{1}\right) \\
& \left.+g_{6}(\phi) \sqrt{n} a(2 N)^{1 / 2} I_{1} \sin \left(\Omega-\Omega_{1}\right)\right]
\end{aligned}
$$

and

$$
\begin{array}{ll}
-\varpi & M=\frac{1}{2} n a^{2} e^{2} \\
-\Omega & N=\frac{1}{2} n a^{2} I^{2}
\end{array}
$$

is an approximate canonical set of coordinates and conjugate momenta for the secular Hamiltonian (i.e. the Hamiltonian obtained after the elimination of the $\phi$-dependence).

\subsubsection{Adiabatic approximation}

By construction of the Hamiltonian of the averaged problem (Eq. (23)) the variables $(-\varpi, M)$ and $(-\Omega, N)$ vary on a timescale $\mathcal{O}\left(\mu_{1}^{-1 / 2} T_{1: 1}\right)$ and therefore are much slower than $\theta$, the angle variable of the integrable part $H_{0}$. The general procedure for dealing with this type of quasiintegrable systems involves a canonical transformation of variables such that the new Hamiltonian does not depend on the fast angle and thus the associated action is an adiabatic invariant (Lichtenberg \& Lieberman 1983).
To first-order in the small parameter $\sqrt{\mu_{1}}$, the transformed Hamiltonian is

$\bar{H}=H_{0}+\sqrt{\mu_{1}}<H_{1}>_{\theta}$

where

$<H_{1}>_{\theta}=\frac{1}{2 \pi} \int_{0}^{2 \pi} H_{1} \mathrm{~d} \theta=\frac{1}{T_{1: 1}} \oint H_{1} \frac{\mathrm{d} \phi}{\dot{\phi}}$.

Now from Eq. (8)

$\frac{1}{T_{1: 1}} \oint \frac{\delta a}{a_{1}} \frac{\mathrm{d} \phi}{\dot{\phi}}=\mathcal{O}\left(\mu_{1}\right)$

hence we obtain the transformed Hamiltonian

$\bar{H}=H_{0}-\bar{X}$

with

$$
\begin{aligned}
\bar{X}= & \mu_{1} n_{1}\left[\bar{g}_{1}[l]\left(h^{2}+k^{2}\right)+\bar{g}_{4}[l]\left(p^{2}+q^{2}\right)\right. \\
& +\bar{g}_{2}[l] \sqrt{n_{1}} a_{1} e_{1}\left(h \sin \varpi_{1}+k \cos \varpi_{1}\right) \\
& +\bar{g}_{3}[l] \sqrt{n_{1}} a_{1} e_{1}\left(h \cos \varpi_{1}-k \sin \varpi_{1}\right) \\
& +\bar{g}_{5}[l] \sqrt{n_{1}} a_{1} I_{1}\left(p \sin \Omega_{1}+q \cos \Omega_{1}\right) \\
& \left.+\bar{g}_{6}[l] \sqrt{n_{1}} a_{1} I_{1}\left(p \cos \Omega_{1}-q \sin \Omega_{1}\right)\right]
\end{aligned}
$$

where $(k, h)$ and $(q, p)$ are defined as

$$
\begin{aligned}
k & =\sqrt{n_{1}} a_{1} e \cos \varpi & & h=\sqrt{n_{1}} a_{1} e \sin \varpi \\
q & =\sqrt{n_{1}} a_{1} I \cos \Omega & & p=\sqrt{n_{1}} a_{1} I \sin \Omega .
\end{aligned}
$$

Moreover,

$g_{i}[l]=\frac{1}{T_{1: 1}} \oint g_{i}(\phi) \frac{\mathrm{d} \phi}{\dot{\phi}}$

where $l$ is a parameter that characterises the size of the tadpole or horseshoe orbit which we define as

$l=\pi / 3-\phi_{\min }$

and $\phi_{\min }$ is the minimal distance to $m_{1}$ obtained by solving Eq. (12) with $\dot{\phi}=0$.

From Paper I we recall that $\bar{g}_{5}[l]=-2 \bar{g}_{4}[l]$ and $\bar{g}_{6}[l]=0$; then we write ${ }^{4}$

$$
\begin{aligned}
2 \bar{g}_{4}[l] \mu_{1} n_{1} & =\Gamma[l] \\
2 \bar{g}_{1}[l] \mu_{1} n_{1} & =\gamma[l] \\
\frac{\bar{g}_{2}[l]}{2 \bar{g}_{1}[l]} & =-c[l] \cos b[l] \\
\frac{\bar{g}_{3}[l]}{2 \bar{g}_{1}[l]} & =-c[l] \sin b[l] .
\end{aligned}
$$

We also note that from Eq. (14) we have

$$
a_{0}[l]=\sqrt{\frac{8}{3} \mu_{1}\left(f(\pi / 3-l)-\frac{3}{2}\right)}
$$

and we refer to Paper I for plots of $\Gamma[l], \gamma[l], c[l]$ and $b[l]$ as functions of $a_{0}[l]$.

${ }^{4}$ There is an obvious error in the definition of $c[l]$ in Paper I (Eq. (36)). This only takes the unit value when $l=0$ and when $l \rightarrow \infty$, as can easily be confirmed in Fig. 1b from the same paper. 


\subsubsection{Secular solution}

We now write Hamilton's equations for $(k, h)$ as

$$
\begin{aligned}
\dot{k}+\mathrm{i} \dot{h} & =-\frac{\partial \bar{X}}{\partial h}+\mathrm{i} \frac{\partial \bar{X}}{\partial k} \\
& =\mathrm{i} \gamma[l](k+\mathrm{i} h)-\mathrm{i} \gamma[l] \sqrt{n_{1}} a_{1} c[l] e_{1} \exp \left[\mathrm{i}\left(\varpi_{1}+b[l]\right)\right]
\end{aligned}
$$

which imply the solution for $(e, \varpi)$

$$
\begin{aligned}
e \exp [\mathrm{i} \varpi]= & e_{\mathrm{p}} \exp [\mathrm{i}(\gamma[l] t+\chi)] \\
& +c[l] e_{1} \exp \left[\mathrm{i}\left(\varpi_{1}+b[l]\right)\right]
\end{aligned}
$$

i.e. composed of a proper term (proper eccentricity $e_{\mathrm{p}}$ and proper precession frequency $\gamma[l]$ ) and a forced term (forced eccentricity $c[l] e_{1}$ and forced periapse $\left.b[l]\right)$.

Similarly, we write Hamilton's equations for $(q, p)$ as

$$
\begin{aligned}
\dot{q}+\mathrm{i} \dot{p} & =-\frac{\partial \bar{X}}{\partial p}+\mathrm{i} \frac{\partial \bar{X}}{\partial q} \\
& =\mathrm{i} \Gamma[l](q+\mathrm{i} p)-\mathrm{i} \Gamma[l] \sqrt{n_{1}} a_{1} I_{1} \exp \left[\mathrm{i} \Omega_{1}\right]
\end{aligned}
$$

which imply the solution for $(I, \Omega)$

$$
I \exp [\mathrm{i} \Omega]=I_{\mathrm{p}} \exp [\mathrm{i}(\Gamma[l] t+\Xi)]+I_{1} \exp \left[\mathrm{i} \Omega_{1}\right]
$$

i.e. composed of a proper term (proper inclination $I_{\mathrm{p}}$ and proper precession frequency $\Gamma[l])$ and a forced term $I_{1}$ (note, however, that if we choose the orbital plane of $m_{\mathrm{c}}$ and $m_{1}$ as reference, then this forced term disappears).

\subsection{The effect of an oblate planet}

The Hamiltonian of the averaged problem has now six degrees of freedom (cf. Sect. 2.2) i.e. two more than in the restricted three-body case. However, we can eliminate these extra degrees of freedom by making a canonical transformation to the following variables

$$
\begin{array}{ll}
-\varpi_{\mathrm{r}}=-\varpi-\lambda_{\mathrm{g} 1} & M \\
-\Omega_{\mathrm{r}}=-\Omega-\lambda_{\mathrm{f} 1} & N \\
\lambda_{\mathrm{g} 1} & \tilde{\Lambda}_{\mathrm{g} 1}=M+\Lambda_{\mathrm{g} 1} \\
\lambda_{\mathrm{f} 1} & \tilde{\Lambda}_{\mathrm{f} 1}=N+\Lambda_{\mathrm{f} 1} .
\end{array}
$$

The transformed first-order part of the Hamiltonian (Eq. (32)) is then

$$
\begin{aligned}
\sqrt{\mu_{1}} \tilde{H}_{1}= & -\beta_{1}\left(\tilde{\Lambda}_{\mathrm{g} 1}-\tilde{\Lambda}_{\mathrm{f} 1}\right)+\mu_{1} n_{1}^{2} a_{1}^{2} F(\phi) \frac{\delta a}{a_{1}} \\
& -X-Y
\end{aligned}
$$

with

$$
\begin{aligned}
X= & \mu_{1} n\left[g_{1}(\phi) 2 M+g_{2}(\phi) \sqrt{n} a(2 M)^{1 / 2} e_{1} \cos \varpi_{\mathrm{r}}\right. \\
& +g_{3}(\phi) \sqrt{n} a(2 M)^{1 / 2} e_{1} \sin \varpi_{\mathrm{r}} \\
& +g_{4}(\phi) 2 N+g_{5}(\phi) \sqrt{n} a(2 N)^{1 / 2} I_{1} \cos \Omega_{\mathrm{r}} \\
& \left.+g_{6}(\phi) \sqrt{n} a(2 N)^{1 / 2} I_{1} \sin \Omega_{\mathrm{r}}\right]
\end{aligned}
$$

and

$$
Y=\beta_{1}(M-N)\left(1+\frac{\delta a}{a_{1}}\right)^{-7 / 2}-\beta_{1}(M-N) .
$$

\subsubsection{Adiabatic approximation}

The first term on the right hand side of Eq. (63) can be dropped as there is no explicit dependence on $\lambda_{\mathrm{g} 1}$ or $\lambda_{\mathrm{f} 1}$. Hence, Eq. (63) has the same form as Eq. (42), except for the term $Y$. This introduces the relative precession frequencies

$\frac{\partial Y}{\partial M}=-\frac{\partial Y}{\partial N} \approx-\frac{7}{2} \beta_{1} \frac{\delta a}{a_{1}}=\mathcal{O}\left(\beta_{1} \sqrt{\mu_{1}}\right)$.

As in general $\beta_{1} \ll n_{1}$, the variables $\left(-\varpi_{\mathrm{r}}, M\right)$ and $\left(-\Omega_{\mathrm{r}}, N\right)$ still vary on a time-scale much longer than the co-orbital period $T_{1: 1}=\mathcal{O}\left(n_{1} \sqrt{\mu_{1}}\right)$. We can then follow the procedure described previously, obtaining an Hamiltonian as defined by Eqs. (45) and (46), i.e.

$\bar{H}=H_{0}-\bar{X}-\bar{Y}$

with

$$
\begin{aligned}
\bar{X}= & \mu_{1} n_{1}\left[\bar{g}_{1}[l]\left(h_{\mathrm{r}}^{2}+k_{\mathrm{r}}^{2}\right)+\bar{g}_{4}[l]\left(p_{\mathrm{r}}^{2}+q_{\mathrm{r}}^{2}\right)\right. \\
& +\bar{g}_{2}[l] \sqrt{n_{1}} a_{1} e_{1} k_{\mathrm{r}}+\bar{g}_{3}[l] \sqrt{n_{1}} a_{1} e_{1} h_{\mathrm{r}} \\
& \left.+\bar{g}_{5}[l] \sqrt{n_{1}} a_{1} I_{1} q_{\mathrm{r}}+\bar{g}_{6}[l] \sqrt{n_{1}} a_{1} I_{1} p_{\mathrm{r}}\right]
\end{aligned}
$$

and

$\bar{Y}=\frac{1}{2} \Delta \bar{\beta}\left[\left(h_{\mathrm{r}}^{2}+k_{\mathrm{r}}^{2}\right)-\left(p_{\mathrm{r}}^{2}+q_{\mathrm{r}}^{2}\right)\right]$

where $\left(k_{\mathrm{r}}, h_{\mathrm{r}}\right)$ and $\left(q_{\mathrm{r}}, p_{\mathrm{r}}\right)$ are defined as

$$
\begin{array}{ll}
k_{\mathrm{r}}=\sqrt{n_{1}} a_{1} e \cos \varpi_{\mathrm{r}} & h_{\mathrm{r}}=\sqrt{n_{1}} a_{1} e \sin \varpi_{\mathrm{r}} \\
q_{\mathrm{r}}=\sqrt{n_{1}} a_{1} I \cos \Omega_{\mathrm{r}} & p_{\mathrm{r}}=\sqrt{n_{1}} a_{1} I \sin \Omega_{\mathrm{r}}
\end{array}
$$

and from Eq. (30)

$\Delta \bar{\beta}=-\beta_{1}+\frac{\beta_{1}}{T_{1: 1}} \oint\left(1+\frac{\delta a}{a_{1}}\right)^{-7 / 2} \frac{\mathrm{d} \phi}{\dot{\phi}}=\mathcal{O}\left(\beta_{1} \mu_{1}\right)$.

\subsubsection{Secular solution}

Hamilton's equations for $\left(k_{\mathrm{r}}, h_{\mathrm{r}}\right)$ are

$$
\begin{aligned}
& \dot{k}_{\mathrm{r}}+\mathrm{i} \dot{h}_{\mathrm{r}}=\left(-\frac{\partial}{\partial h_{\mathrm{r}}}+\mathrm{i} \frac{\partial}{\partial k_{\mathrm{r}}}\right)(\bar{X}+\bar{Y})= \\
& \mathrm{i}(\gamma[l]+\Delta \bar{\beta})\left(k_{\mathrm{r}}+\mathrm{i} h_{\mathrm{r}}\right)-\mathrm{i} \gamma[l] \sqrt{n_{1}} a_{1} c[l] e_{1} \exp [\mathrm{i} b[l]]
\end{aligned}
$$

and therefore the solution for $\left(e, \varpi_{\mathrm{r}}\right)$ is

$$
\begin{aligned}
e \exp \left[\mathrm{i} \varpi_{\mathrm{r}}\right]= & e_{\mathrm{p}} \exp [\mathrm{i}((\gamma[l]+\Delta \bar{\beta}) t+\chi)] \\
& +\frac{\gamma[l] c[l] e_{1} \exp [\mathrm{i} b[l]]}{\gamma[l]+\Delta \bar{\beta}} .
\end{aligned}
$$

Similarly, Hamilton's equations for $\left(q_{\mathrm{r}}, p_{\mathrm{r}}\right)$ are

$$
\begin{aligned}
\dot{q}_{\mathrm{r}}+\mathrm{i} \dot{p}_{\mathrm{r}} & =\left(-\frac{\partial}{\partial p_{\mathrm{r}}}+\mathrm{i} \frac{\partial}{\partial q_{\mathrm{r}}}\right)(\bar{X}+\bar{Y}) \\
& =\mathrm{i}(\Gamma[l]-\Delta \bar{\beta})\left(q_{\mathrm{r}}+\mathrm{i} p_{\mathrm{r}}\right)-\mathrm{i} \Gamma[l] \sqrt{n_{1}} a_{1} I_{1}
\end{aligned}
$$

and therefore the solution for $\left(I, \Omega_{\mathrm{r}}\right)$ is

$$
\begin{aligned}
I \exp \left[\mathrm{i} \Omega_{\mathrm{r}}\right]= & I_{\mathrm{p}} \exp [\mathrm{i}((\Gamma[l]-\Delta \bar{\beta}) t+\Xi)] \\
& +\frac{\Gamma[l] I_{1}}{\Gamma[l]-\Delta \bar{\beta}} .
\end{aligned}
$$


From Eqs. (73) and (75) we see that the oblateness term, $\Delta \bar{\beta}>0$, leads to a decrease in the secular precession periods and the magnitude of the forced terms. However, this effect is only significant if $\Delta \bar{\beta} \sim \gamma$ (or $-\Gamma$ ). As $\gamma \geq 3.375 n_{1} \mu_{1}$ (see Fig. 1a in Paper I) and in general $\beta_{1} \ll n_{1}$, one can largely neglect the term $\Delta \bar{\beta}$ in that which concerns the evolution of the eccentricity; in this case the secular solution reduces to Eq. (59). On the other hand, very small amplitude tadpole orbits can have $\Gamma \sim-\beta_{1} \mu_{1}$ (see Fig. 1d in Paper I) and thus the effect of the term $\Delta \bar{\beta}$ on the evolution of the inclination can be visible.

\subsection{The effect of additional massive bodies}

The Hamiltonian of the averaged problem has $2 \times N+$ 4 degrees of freedom (cf. Sect. 2.3). In order to reduce the number of degrees of freedom we perform a canonical transformation to the following variables

$$
\begin{array}{ll}
-\varpi_{\mathrm{r}}=-\varpi-\lambda_{\mathrm{g} k} & M \\
-\Omega_{\mathrm{r}}=-\Omega-\lambda_{\mathrm{f} k} & N \\
\lambda_{\mathrm{g} k} & \tilde{\Lambda}_{\mathrm{g} k}=M+\Lambda_{\mathrm{g} k} \\
\lambda_{\mathrm{f} k} & \tilde{\Lambda}_{\mathrm{f} k}=N+\Lambda_{\mathrm{f} k} .
\end{array}
$$

We also perform $N-1$ canonical transformations of the same type to the following variables

$$
\begin{array}{ll}
\check{\lambda}_{\mathrm{g} i}=\lambda_{\mathrm{g} i}-\lambda_{\mathrm{g} k} & \Lambda_{\mathrm{g} i} \\
\check{\lambda}_{\mathrm{f} i}=\lambda_{\mathrm{f} i}-\lambda_{\mathrm{f} k} & \check{\Lambda}_{\mathrm{f} i} \\
\lambda_{\mathrm{g} k} & \check{\Lambda}_{\mathrm{g} k}=\sum_{i \neq k} \Lambda_{\mathrm{g} i}+\tilde{\Lambda}_{\mathrm{g} k} \\
\lambda_{\mathrm{f} k} & \check{\Lambda}_{\mathrm{f} k}=\sum_{i \neq k} \Lambda_{\mathrm{f} i}+\tilde{\Lambda}_{\mathrm{f} k} .
\end{array}
$$

Then, the transformed first-order part of the Hamiltonian (Eq. (40)) is

$$
\begin{aligned}
\sqrt{\mu_{k}} \check{H}_{1}= & -\check{\Lambda}_{\mathrm{g} k} g_{k}-\check{\Lambda}_{\mathrm{f} k} f_{k} \\
& -\sum_{i \neq k} \Lambda_{\mathrm{g} i}\left(g_{i}-g_{k}\right)-\sum_{i \neq k} \Lambda_{\mathrm{f} i}\left(f_{i}-f_{k}\right) \\
& +\mu_{k} n_{k}^{2} a_{k}^{2} F(\phi) \frac{\delta a}{a_{k}}-X-Y
\end{aligned}
$$

with

$$
\begin{aligned}
X= & \mu_{k} n\left[g_{1}(\phi) 2 M+g_{4}(\phi) 2 N\right. \\
& +g_{2}(\phi) \sqrt{n} a(2 M)^{1 / 2} \sum_{i} e_{k, i} \cos \left(\varpi_{\mathrm{r}}+\check{\lambda}_{\mathrm{g} i}\right) \\
& +g_{3}(\phi) \sqrt{n} a(2 M)^{1 / 2} \sum_{i} e_{k, i} \sin \left(\varpi_{\mathrm{r}}+\check{\lambda}_{\mathrm{g} i}\right) \\
& +g_{5}(\phi) \sqrt{n} a(2 N)^{1 / 2} \sum_{i} I_{k, i} \cos \left(\Omega_{\mathrm{r}}+\check{\lambda}_{\mathrm{f} i}\right) \\
& \left.+g_{6}(\phi) \sqrt{n} a(2 N)^{1 / 2} \sum_{i} I_{k, i} \sin \left(\Omega_{\mathrm{r}}+\check{\lambda}_{\mathrm{f} i}\right)\right]
\end{aligned}
$$

and

$$
\begin{aligned}
Y= & \left(A_{k}-g_{k}\right) M+\left(B_{k}-f_{k}\right) N \\
& +\sum_{j \neq k} A_{j} \sqrt{n} a(2 M)^{1 / 2} \sum_{i} e_{j, i} \cos \left(\varpi_{\mathrm{r}}+\check{\lambda}_{\mathrm{g} i}\right) \\
& +\sum_{j \neq k} B_{j} \sqrt{n} a(2 N)^{1 / 2} \sum_{i} I_{j, i} \cos \left(\Omega_{\mathrm{r}}+\check{\lambda}_{\mathrm{f} i}\right) .
\end{aligned}
$$

And the first two terms on the right hand side of Eq. (78) can be dropped as there is no explicit dependence on $\lambda_{\mathrm{g} k}$ or $\lambda_{\mathrm{f} k}$.

\subsubsection{Secular resonances}

We now assume that the perturbers move on circular and co-planar orbits, in which case the terms in Eq. (78) depending on $\left(\check{\lambda}_{\mathrm{g} i}, \Lambda_{\mathrm{g} i}\right)$ and $\left(\check{\lambda}_{\mathrm{f} i}, \Lambda_{\mathrm{f} i}\right)$ also disappear and the Hamiltonian reduces to

$$
\begin{aligned}
H= & H_{0}+\mu_{k} n_{k}^{2} a_{k}^{2} F(\phi) \frac{\delta a}{a_{k}} \\
& -\left(2 g_{1}(\phi) \mu_{k} n+A_{k}-g_{k}\right) M \\
& -\left(2 g_{4}(\phi) \mu_{k} n+B_{k}-f_{k}\right) N .
\end{aligned}
$$

Equation (81) represents a one-degree of freedom (hence integrable) system depending on the parameters $M$ and $N$. Therefore, we can perform a canonical transformation to action-angle variables which eliminates $\phi$ from the Hamiltonian (Lichtenberg \& Lieberman 1983). To firstorder in the small parameter $\sqrt{\mu_{k}}$, this is accomplished by averaging over the angle variable of the zero-order term $H_{0}$, which is equivalent to averaging over the co-orbital period $T_{1: 1}$. The transformed Hamiltonian is

$\bar{H}=H_{0}-\left(\gamma_{k}[l]+\bar{A}_{k}-g_{k}\right) M-\left(\Gamma_{k}[l]+\bar{B}_{k}-f_{k}\right) N$

which has proper frequencies

$\dot{\varpi}_{\mathrm{r}}=-\frac{\partial \bar{H}}{\partial M}=\gamma_{k}[l]+\bar{A}_{k}-g_{k}$
$\dot{\Omega}_{\mathrm{r}}=-\frac{\partial \bar{H}}{\partial N}=\Gamma_{k}[l]+\bar{B}_{k}-f_{k}$

where $\gamma_{k}[l]=2 \bar{g}_{1}[l] \mu_{k} n_{k}, \Gamma_{k}[l]=2 \bar{g}_{4}[l] \mu_{k} n_{k}$; and from Eq. (38)

$$
\begin{aligned}
& \bar{A}_{k}=\frac{1}{T_{1: 1}} \oint A_{k} \frac{\mathrm{d} \phi}{\dot{\phi}}=A_{k, k}\left(1+\mathcal{O}\left(\mu_{k}\right)\right) \\
& \bar{B}_{k}=\frac{1}{T_{1: 1}} \oint B_{k} \frac{\mathrm{d} \phi}{\dot{\phi}}=B_{k, k}\left(1+\mathcal{O}\left(\mu_{k}\right)\right) .
\end{aligned}
$$

Secular resonances involving the pericentres (or nodes) occur when $\dot{\varpi}_{\mathrm{r}}\left(\right.$ or $\left.\dot{\Omega}_{\mathrm{r}}\right)$ is equal to a forcing frequency $g_{i}-g_{k}$ (or $f_{i}-f_{k}$ ), i.e. when

$$
\begin{gathered}
\gamma_{k}[l]+\bar{A}_{k}=g_{i} \\
\Gamma_{k}[l]+\bar{B}_{k}=f_{i} .
\end{gathered}
$$

Note that the proper frequencies of precession of the Trojan orbit are in fact $\dot{\varpi}=\dot{\varpi}_{\mathrm{r}}+g_{k}$ and $\dot{\Omega}=\dot{\Omega}_{\mathrm{r}}+f_{k}$ which coincide respectively with the left hand side of Eq. (87) and Eq. (88). Moreover, the terms $\gamma_{k}$ (and $\Gamma_{k}$ ) and the terms $\bar{A}_{k}$ (and $\bar{B}_{k}$ ) are respectively the contribution from the mass $m_{k}$ and the contribution from the additional massive bodies $m_{j}$ (where $j \neq k$ ). 


\subsubsection{Adiabatic approximation}

We will now assume that not only the variables $\left(-\varpi_{\mathrm{r}}, M\right)$ and $\left(-\Omega_{\mathrm{r}}, N\right)$ but also the angles $\check{\lambda}_{\mathrm{g} i}$ and $\check{\lambda}_{\mathrm{f} i}$ vary on a time-scale much longer than the co-orbital period $T_{1: 1}$. Note that while the first assumption will in general be true, the same does not necessarily apply to the second assumption. When the co-orbital frequency is comparable to one of the forcing frequencies, a low order resonance can occur in which case the adiabatic approximation does not provide a good description of the system. Nevertheless, if we ignore this possibility then we can apply Eqs. (45) and (46) to obtain the transformed Hamiltonian

$$
\begin{aligned}
\bar{H}= & H_{0}-\sum_{i \neq k} \Lambda_{\mathrm{g} i}\left(g_{i}-g_{k}\right)-\sum_{i \neq k} \Lambda_{\mathrm{f} i}\left(f_{i}-f_{k}\right) \\
& -\bar{X}-\bar{Y}
\end{aligned}
$$

with

$$
\begin{aligned}
\bar{X}= & \mu_{k} n_{k}\left[\bar{g}_{1}[l]\left(h_{\mathrm{r}}^{2}+k_{\mathrm{r}}^{2}\right)+\bar{g}_{4}[l]\left(p_{\mathrm{r}}^{2}+q_{\mathrm{r}}^{2}\right)\right. \\
& +\bar{g}_{2}[l] \sqrt{n_{k}} a_{k} \sum_{i} e_{k, i}\left(k_{\mathrm{r}} \cos \check{\lambda}_{\mathrm{g} i}-h_{\mathrm{r}} \sin \check{\lambda}_{\mathrm{g} i}\right) \\
& +\bar{g}_{3}[l] \sqrt{n_{k}} a_{k} \sum_{i} e_{k, i}\left(h_{\mathrm{r}} \cos \check{\lambda}_{\mathrm{g} i}+k_{\mathrm{r}} \sin \check{\lambda}_{\mathrm{g} i}\right) \\
& +\bar{g}_{5}[l] \sqrt{n_{k}} a_{k} \sum_{i} I_{k, i}\left(q_{\mathrm{r}} \cos \check{\lambda}_{\mathrm{f} i}-p_{\mathrm{r}} \sin \check{\lambda}_{\mathrm{f} i}\right) \\
& \left.+\bar{g}_{6}[l] \sqrt{n_{k}} a_{k} \sum_{i} I_{k, i}\left(p_{\mathrm{r}} \cos \check{\lambda}_{\mathrm{f} i}+q_{\mathrm{r}} \sin \check{\lambda}_{\mathrm{f} i}\right)\right]
\end{aligned}
$$

and

$$
\begin{aligned}
\bar{Y}= & \frac{1}{2}\left(\bar{A}_{k}-g_{k}\right)\left(h_{\mathrm{r}}^{2}+k_{\mathrm{r}}^{2}\right)+\frac{1}{2}\left(\bar{B}_{k}-f_{k}\right)\left(p_{\mathrm{r}}^{2}+q_{\mathrm{r}}^{2}\right) \\
& +\sum_{j \neq k} \bar{A}_{j} \sqrt{n_{j}} a_{j} \sum_{i} e_{j, i}\left(k_{\mathrm{r}} \cos \check{\lambda}_{\mathrm{g} i}-h_{\mathrm{r}} \sin \check{\lambda}_{\mathrm{g} i}\right) \\
& +\sum_{j \neq k} \bar{B}_{j} \sqrt{n_{j}} a_{j} \sum_{i} I_{j, i}\left(q_{\mathrm{r}} \cos \check{\lambda}_{\mathrm{f} i}-p_{\mathrm{r}} \sin \check{\lambda}_{\mathrm{f} i}\right)
\end{aligned}
$$

where $\left(k_{\mathrm{r}}, h_{\mathrm{r}}\right)$ and $\left(q_{\mathrm{r}}, p_{\mathrm{r}}\right)$ are defined as

$$
\begin{aligned}
k_{\mathrm{r}}+\mathrm{i} h_{\mathrm{r}} & =\sqrt{n_{k}} a_{k} e \exp \left[\mathrm{i} \varpi_{\mathrm{r}}\right] \\
q_{\mathrm{r}}+\mathrm{i} p_{\mathrm{r}} & =\sqrt{n_{k}} a_{k} I \exp \left[\mathrm{i} \Omega_{\mathrm{r}}\right]
\end{aligned}
$$

and from Eq. (38)

$$
\begin{aligned}
& \bar{A}_{j}=\frac{1}{T_{1: 1}} \oint A_{j} \frac{\mathrm{d} \phi}{\dot{\phi}}=A_{k, j}\left(1+\mathcal{O}\left(\mu_{k}\right)\right) \\
& \bar{B}_{j}=\frac{1}{T_{1: 1}} \oint B_{j} \frac{\mathrm{d} \phi}{\dot{\phi}}=B_{k, j}\left(1+\mathcal{O}\left(\mu_{k}\right)\right) .
\end{aligned}
$$

\subsubsection{Secular solution}

The evolution of $z=\sqrt{n_{k}} a_{k} e \exp [\mathrm{i} \varpi]$ is described by

$$
\begin{aligned}
& \left(\dot{z}-\mathrm{i} g_{k} z\right) \exp \left[\mathrm{i} \lambda_{\mathrm{g} k}\right]=\left(-\frac{\partial}{\partial h_{\mathrm{r}}}+\mathrm{i} \frac{\partial}{\partial k_{\mathrm{r}}}\right)(\bar{X}+\bar{Y})= \\
& \mathrm{i}\left(\left(\gamma_{k}[l]+\bar{A}_{k}-g_{k}\right) z-\gamma_{k}[l] \sqrt{n_{k}} a_{k} c[l] e_{k} \exp \left[\mathrm{i}\left(\varpi_{k}+b[l]\right)\right]\right. \\
& \left.+\sum_{j \neq k} \bar{A}_{j} \sqrt{n_{j}} a_{j} e_{j} \exp \left[\mathrm{i} \varpi_{j}\right]\right) \exp \left[\mathrm{i} \lambda_{\mathrm{g} k}\right]
\end{aligned}
$$

which is the equation of a forced harmonic oscillator with proper frequency $\gamma_{k}[l]+\bar{A}_{k}$ and forcing frequencies $g_{i}$; hence the solution for $(e, \varpi)$ is

$$
\begin{aligned}
& e \exp [\mathrm{i} \varpi]=e_{\mathrm{p}} \exp \left[\mathrm{i}\left(\left(\gamma_{k}[l]+\bar{A}_{k}\right) t+\chi\right)\right] \\
& +\sum_{i} \frac{\gamma_{k}[l] c[l] e_{k, i}}{\gamma_{k}[l]+\bar{A}_{k}-g_{i}} \exp \left[\mathrm{i}\left(g_{i} t+\chi_{i}+b[l]\right)\right] \\
& -\sum_{i} \frac{\sum_{j \neq k} \bar{A}_{j} e_{j, i}}{\gamma_{k}[l]+\bar{A}_{k}-g_{i}} \exp \left[\mathrm{i}\left(g_{i} t+\chi_{i}\right)\right] .
\end{aligned}
$$

The evolution of $Z=\sqrt{n_{k}} a_{k} I \exp [\mathrm{i} \Omega]$ is described by

$$
\begin{aligned}
& \left(\dot{Z}-\mathrm{i} f_{k} Z\right) \exp \left[\mathrm{i} \lambda_{\mathrm{f} k}\right]=\left(-\frac{\partial}{\partial p_{\mathrm{r}}}+\mathrm{i} \frac{\partial}{\partial q_{\mathrm{r}}}\right)(\bar{X}+\bar{Y}) \\
& =\mathrm{i}\left(\left(\Gamma_{k}[l]+\bar{B}_{k}-f_{k}\right) Z-\Gamma_{k}[l] \sqrt{n_{k}} a_{k} I_{k} \exp \left[\mathrm{i} \Omega_{k}\right]\right. \\
& \left.+\sum_{j \neq k} \bar{B}_{j} \sqrt{n_{j}} a_{j} I_{j} \exp \left[\mathrm{i} \Omega_{j}\right]\right) \exp \left[\mathrm{i} \lambda_{\mathrm{f} k}\right]
\end{aligned}
$$

which is the equation of a forced harmonic oscillator with proper frequency $\Gamma_{k}[l]+\bar{B}_{k}$ and forcing frequencies $f_{i}$; hence the solution for $(I, \Omega)$ is

$$
\begin{aligned}
& I \exp [\mathrm{i} \Omega]=I_{\mathrm{p}} \exp \left[\mathrm{i}\left(\left(\Gamma_{k}[l]+\bar{B}_{k}\right) t+\Xi\right)\right] \\
& +\sum_{i} \frac{\Gamma_{k}[l] I_{k, i}-\sum_{j \neq k} \bar{B}_{j} I_{j, i}}{\Gamma_{k}[l]+\bar{B}_{k}-f_{i}} \exp \left[\mathrm{i}\left(f_{i} t+\Xi_{i}\right)\right] .
\end{aligned}
$$

We now recall from Paper I that $\bar{B}_{j}=\left(1+\mathcal{O}\left(\mu_{k}\right)\right) B_{k, j}$, and that by definition of eigen-values and eigen-vectors, $B_{k, k} I_{k, i}+\sum_{j \neq k} B_{k, j} I_{j, i}=f_{i} I_{k, i}$, so that

$$
\begin{aligned}
I \exp [\mathrm{i} \Omega]= & I_{\mathrm{p}} \exp \left[\mathrm{i}\left(\left(\Gamma_{k}[l]+\bar{B}_{k}\right) t+\Xi\right)\right] \\
& +\sum_{i} I_{k, i} \exp \left[\mathrm{i}\left(f_{i} t+\Xi_{i}\right)\right] \\
& +\sum_{i} \frac{\mathcal{O}\left(\mu_{k} \mu_{i}\right) I_{k, i}}{\Gamma_{k}[l]+\bar{B}_{k}-f_{i}} \exp \left[\mathrm{i}\left(f_{i} t+\Xi_{i}\right)\right]
\end{aligned}
$$

This is to say that when $\Gamma_{k}[l]+\bar{B}_{k}=f_{i}$, the forcing terms in the second-order differential equation that describes the evolution of $I \exp [\mathrm{i} \Omega]$ are smaller than in the non-resonant case by a mass ratio factor. Indeed, one can show that these forcing terms have amplitudes $I_{k, i}\left(\Gamma_{k}[l]+\bar{B}_{k}+f_{i}\right)\left[\left(\Gamma_{k}[l]+\bar{B}_{k}-f_{i}\right)+\mathcal{O}\left(\mu_{k} \mu_{i}\right)\right]$, which reduce to $2 f_{i} I_{k, i} \mathcal{O}\left(\mu_{k} \mu_{i}\right)$ at the exact resonance.

Note that although the secular solution obtained here (Eqs. (97) and (99)) has essentially the same form as that obtained in Paper I, the terms $\bar{A}_{j}$ and $\bar{B}_{j}$ are now defined as averages over $T_{1: 1}$ (which nonetheless coincide, to lowest order, with the quantities defined in Paper I). The basic improvement with respect to Paper I is the correct derivation of the thresholds of validity of the secular solution which as we have seen now simply depends on the validity of the adiabatic approximation. 
Table 1. Uranian satellite system: co-orbital frequencies $\sqrt{(27 / 4) \mu_{i}} n_{i}$ and forcing frequencies $g_{i}-g_{j}$ and $f_{i}-f_{j}$ (units are rads/day). Note that $g_{i}-g_{j}$ and $f_{j}-f_{i}$ are nearly equal; this is due to the fact that $g_{k}$ and $f_{k}$ are respectively the eigenvalues of matrices $A$ and $B$ which by definition have $A_{k k}=-B_{k k}$ and which are also nearly diagonal (the diagonal term is mostly due to the oblateness and therefore $\left.A_{k k}=-B_{k k} \approx \beta\left(a_{k}\right)\right)$

\begin{tabular}{ccccccc}
\hline $\mathrm{i}$ & $\sqrt{(27 / 4) \mu_{i}} n_{i}$ & $g_{1}-g_{i}$ & $g_{2}-g_{i}$ & $g_{3}-g_{i}$ & $g_{4}-g_{i}$ & $g_{5}-g_{i}$ \\
& & $f_{1}-f_{i}$ & $f_{2}-f_{i}$ & $f_{3}-f_{i}$ & $f_{4}-f_{i}$ & $f_{5}-f_{i}$ \\
\hline 1 & $1.110^{-2}$ & 0 & $-7.010^{-4}$ & $-8.510^{-4}$ & $-9.110^{-4}$ & $-9.710^{-4}$ \\
& & 0 & $7.010^{-4}$ & $8.510^{-4}$ & $9.110^{-4}$ & $9.710^{-4}$ \\
\hline 2 & $2.910^{-2}$ & $7.010^{-4}$ & 0 & $-1.510^{-4}$ & $-2.110^{-4}$ & $-2.710^{-4}$ \\
& & $-7.010^{-4}$ & 0 & $1.510^{-4}$ & $2.110^{-4}$ & $2.810^{-4}$ \\
\hline 3 & $1.310^{-2}$ & $8.510^{-4}$ & $1.510^{-4}$ & 0 & $-6.010^{-5}$ & $-1.210^{-4}$ \\
& & $-8.510^{-4}$ & $-1.510^{-4}$ & 0 & $5.410^{-5}$ & $1.210^{-4}$ \\
\hline 4 & $1.110^{-2}$ & $9.110^{-4}$ & $2.110^{-4}$ & $6.010^{-5}$ & 0 & $-6.110^{-5}$ \\
& & $-9.010^{-4}$ & $-2.110^{-4}$ & $-5.410^{-5}$ & 0 & $6.910^{-5}$ \\
\hline 5 & $7.010^{-3}$ & $9.710^{-4}$ & $2.710^{-4}$ & $1.210^{-4}$ & $6.110^{-5}$ & 0 \\
& & $-9.710^{-4}$ & $-2.810^{-4}$ & $-1.210^{-4}$ & $-6.910^{-5}$ & 0 \\
\hline
\end{tabular}

\section{Applications}

\subsection{The uranian satellite system}

We will now apply our secular theory to the case of Trojan orbits associated with the five major uranian satellites. As already noticed in Paper I, this system provides a good testing ground for our theory due to two reasons. First: there are no mean motion resonances amongst these satellites and thus the secular approximation (i.e. the average of the disturbing potential over the mean longitudes) is valid. Second: these satellites have nearly-circular and nearly-equatorial orbits thus satisfying the requirements of our secular theory.

We used the data from Malhotra et al. (1989) to calculate the parameters of the secular theory for the uranian system ${ }^{5}$ and then identified the modes that satisfy Eq. (87) (or Eq. (88)) inside the co-orbital regions of its major satellites. For instance, in order to locate secular resonances involving the pericentres (or nodes) inside the co-orbital region of the mass $m_{k}$, we first plot $\gamma_{k}\left(\text { or } \Gamma_{k}\right)^{6}$ as a function of $a_{0}$ (a parameter that characterizes the size of the tadpole or horseshoe orbit already defined in Eq. (14)) and then identify the intersection with the horizontal lines taken at $g_{i}-\bar{A}_{k}$ (or $\left.f_{i}-\bar{B}_{k}\right)$. We show the location of the secular resonances which occur inside the co-orbital regions of Miranda $\left(m_{1}\right)$, Ariel $\left(m_{2}\right)$, Umbriel $\left(m_{3}\right)$, Titania $\left(m_{4}\right)$ and Oberon $\left(m_{5}\right)$ in Fig. 2 . Note that we can condense the results for the five satellites in one single picture due to the fact that both $\gamma_{k}$ and $\Gamma_{k}$ scale as $\mu_{k} n_{k}$ (see also Paper I).

\footnotetext{
${ }^{5}$ Here, we took into account Uranus' oblate shape by adding the terms $\beta\left(a_{k}\right)$ (recall definition of function $\beta$ in Eq. (26)) to $A_{k k}$ and $-B_{k k}$ (the diagonal terms of matrices $A$ and $-B$ which we introduced in Sect. 2.3).

${ }^{6}$ In our calculations we ignored the contribution $\Delta \bar{\beta}$ (given by Eq. (71)) due to Uranus' oblate shape as in general this is unimportant (cf. last paragraph of Sect. 3.2.2).
}

We also used a Runge-Kutta-Nystron 12th order scheme (Brankin et al. 1987) to integrate the equations of motion of the system consisting of Uranus, its satellites and associated test particles in tadpole or horseshoe orbits. The parameters and initial conditions were again taken from Malhotra et al. (1989), and we incorporated an oblateness potential which takes into account only the dominant zonal harmonic.

In Paper I we showed the result of a numerical integration for a test particle located near the $L_{4}$ point of Titania $\left(m_{4}\right)$, which included the gravitational interaction with Umbriel $\left(m_{3}\right)$ only. We saw that in this case the longterm behaviour of $(e, \varpi)$ is affected by the proximity of a secular resonance involving the mode $g_{3}$ and is in good agreement with the secular solution (Eq. (97)). In fact, we can see from Table 1 that the adiabatic approximation made in Sect. 3.3.2 should be valid in the case of the uranian satellites, as the co-orbital frequencies are much larger than the forcing frequencies.

In Fig. 3 we show the evolution of an orbit located near the $L_{4}$ point of Oberon with $a_{0}=0$. This is very close to the secular resonance involving the mode $g_{4}$ (cf. Fig. 2) and we see that the slow periodic motion of the critical argument $\varpi-g_{4} t$ is correlated with a large amplitude oscillation of the eccentricity. The small amplitude fast oscillation in the eccentricity has the same periodicity as the argument $\varpi-g_{5} t$ and is indeed caused by the forced term due to the 1:1 mean motion resonance with Oberon. There is no apparent threat to the long-term stability of this orbit, as the maximum eccentricity is still very far from the threshold required for close approaches with nearby Titania (i.e. $e=0.25$ ).

In Fig. 4 we show the evolution of a horseshoe orbit associated with Oberon which has $a_{0}=2 \sqrt{\mu_{5}}$. The eccentricity exhibits very irregular behaviour which is probably due to the interaction between two nearby secular resonances (involving the modes $g_{3}$ and $g_{4}$; cf. Fig. 2) as 

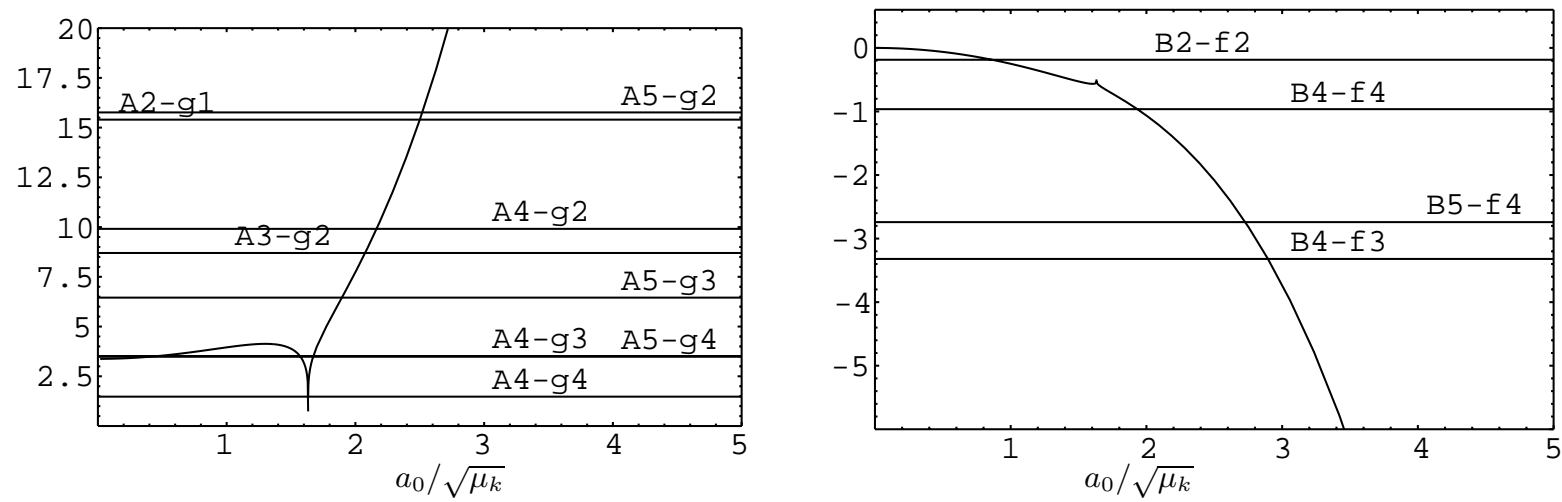

Fig. 2. Location of secular resonances involving a) the pericentres and $\mathbf{b}$ ) the nodes, inside the co-orbital regions of the uranian satellites Miranda $\left(m_{1}\right)$, Ariel $\left(m_{2}\right)$, Umbriel $\left(m_{3}\right)$, Titania $\left(m_{4}\right)$ and Oberon $\left(m_{5}\right)$. Secular resonances associated with the modes $g_{i}$ (or $f_{i}$ ) occur at the locations $a_{0} / \sqrt{\mu_{k}}$ determined by the intersections of the curves $\gamma_{k}$ (or $\Gamma_{k}$ ) with the lines labeled $A_{k}-g_{i}$ (or $B_{k}-f_{i}$ ). Note that the frequencies $\gamma_{k}$ and $\Gamma_{k}$ were divided by $\mu_{k} n_{k}$ in order to be able to condense the results for all the five satellites in one single picture. The singularity in $\gamma_{k}$ and $\Gamma_{k}$ at $a_{0}=\sqrt{(8 / 3) \mu_{1}}$ corresponds to the tadpole-horseshoe separatrix
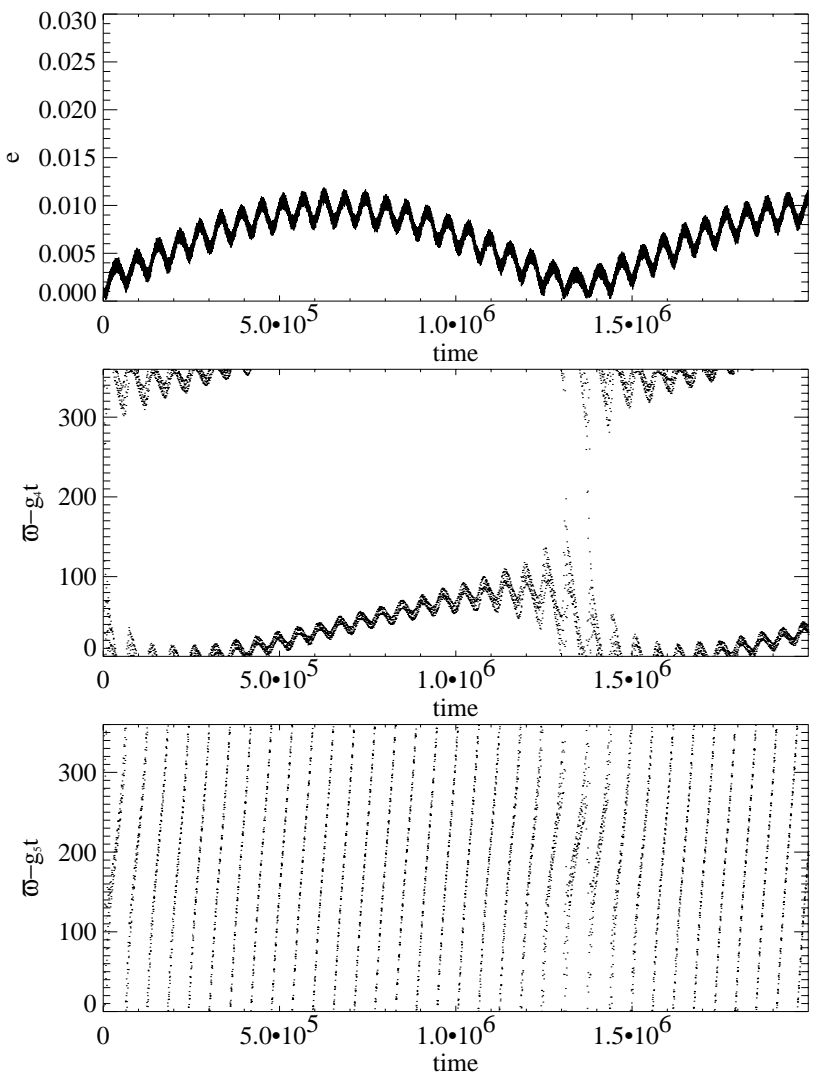

Fig. 3. Evolution of eccentricity (upper figure), critical arguments associated with the modes $g_{4}$ (middle figure) and $g_{5}$ (lower figure) for an orbit located near Oberon's $L_{4}$ point with $a_{0}=0$. Time is in units of Miranda's orbital period

suggested by the behaviour of the associated critical arguments. Due to the overlap of the separatrices of these two secular resonances, the eccentricity diffuses chaotically and can potentially reach stability-threatening values.
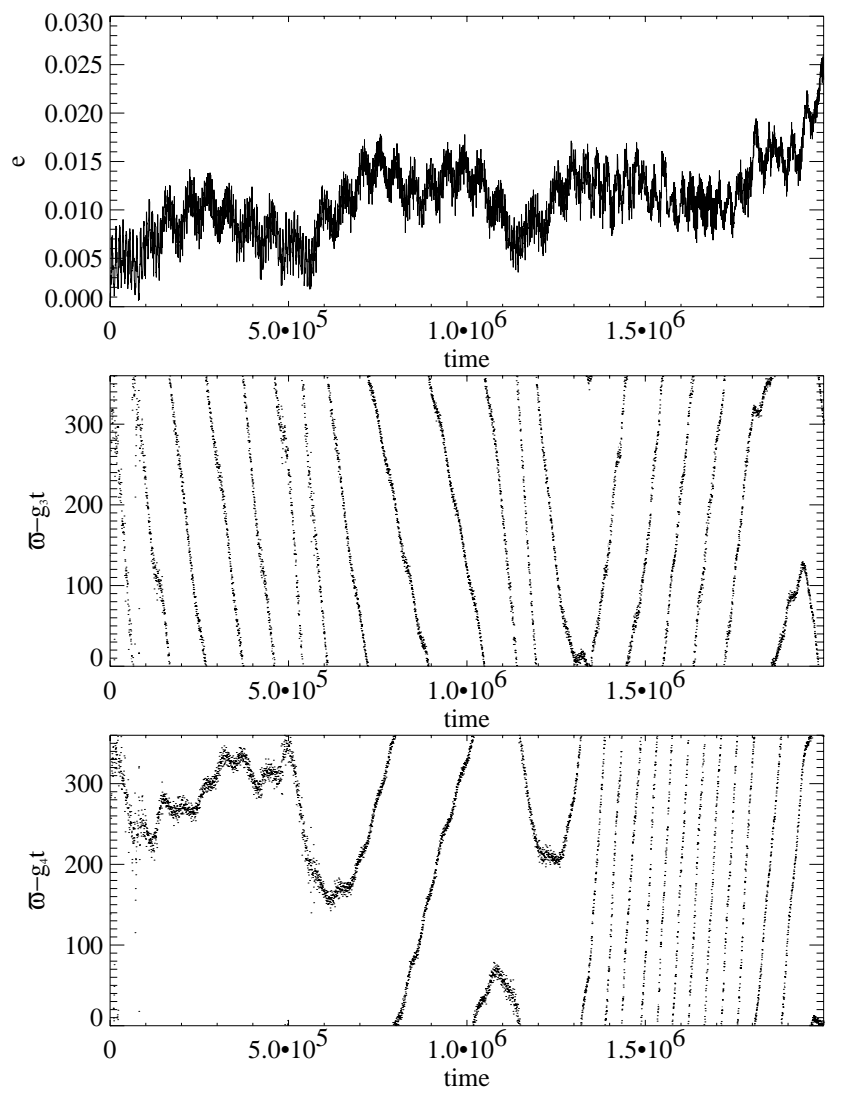

Fig. 4. Evolution of eccentricity (upper figure), critical arguments associated with the modes $g_{3}$ (middle figure) and $g_{4}$ (lower figure) for a horseshoe orbit associated with Oberon with $a_{0}=2 \sqrt{\mu_{5}}$. Time is in units of Miranda's orbital period

We remark here that although our secular theory cannot describe accurately the true behaviour in the very close vicinity of secular resonances (due to the occurrence of singularities in Eqs. (97) and (99) which is an artifact 



Fig. 5. Location of secular resonances involving the pericentres inside the co-orbital regions of the planets. The frequencies shown in the vertical axes are adimensional and correspond to the true frequencies divided by $\mu_{k} n_{k}$. Secular resonances associated with the modes $g_{i}$ occur at the locations $a_{0} / \sqrt{\mu_{k}}$ determined by the intersections of the lines labeled $A_{k}-g_{i}$ with the curves $\gamma_{k}$. The secular resonances involving the modes $g_{1}$ and $g_{2}$ (not shown here as they are likely to be very weak) also occur inside the co-orbital regions of Uranus and Neptune

caused by the truncation of the disturbing potential at degree two in $e$ and $I$ ) or whenever there is overlap of adjacent secular resonances (due to the underlying chaotic nature of the phase space), it is still very useful in the sense that it allows us to obtain the location of these secular resonances, whose dynamical effect can always be subsequently investigated with numerical integrations.

\subsection{Our planetary system}

We also applied our secular theory to the system consisting of the eight planets, Mercury to Neptune, using values for the eigen-frequencies of the secular system from Brouwer \& Van Woerkom (1950). As these were calculated taking in account the effect of the 2:5 near commensurability between the orbital periods of Jupiter and Saturn, the secular system is characterised by ten eigen-frequencies $g_{i}$ (with $g_{9}=2 g_{5}-g_{6}$ and $g_{10}=2 g_{6}-g_{5}$ ) and eight eigen-frequencies $f_{i}$. We then used these in order to obtain the locations of secular resonances inside the planetary co-orbital regions ${ }^{7}$ condensed in the six panels of Figs. 5 and 6.

Figure $5 \mathrm{f}$ proves that the secular resonances involving the modes $g_{6}$ and $g_{10}$ can affect Saturn's tadpole orbits which is in agreement with recent numerical integrations by Marzari \& Scholl (2000). In particular, these latter authors suggest that the mixed secular resonance involving

\footnotetext{
7 The use of more accurate values for the eigen-frequencies from Laskar (1988) does not cause any significant alteration in the location of these secular resonances.
} 
a


Fig. 6. Location of secular resonances involving the nodes inside the co-orbital regions of the planets. The frequencies shown in the vertical axes are adimensional and correspond to the true frequencies divided by $\mu_{k} n_{k}$. Secular resonances associated with the modes $f_{i}$ occur at the locations $a_{0} / \sqrt{\mu_{k}}$ determined by the intersections of the lines labeled $B_{k}-f_{i}$ with the curves $\Gamma_{k}$

the mode $g_{10}$ is the major factor responsible for the destabilization of tadpole orbits associated with Saturn.

In Fig. $6 \mathrm{~b}$ we see that the secular resonances involving the modes $f_{3}$ and $f_{4}$ occur very close to each other inside Venus' horseshoe region, thus suggesting their possible overlap. In fact, the numerical integrations of Michel (1997) showed that a clone of asteroid (4660) Nereus becomes, at some stage in its life, a Venus' horseshoe orbit which exhibits chaotic diffusion of the inclination caused by the overlap of these two secular resonances.

From Fig. 6d and as already mentioned in Paper I, we see that the secular resonance involving the mode $f_{6}$ occurs within Jupiter's tadpole region. Recent numerical integrations by Marzari \& Scholl (2000) seem to support earlier suggestions by Yoder (1979) and Milani (1994) concerning the important role played by this secular resonance in the dynamical shaping of the Trojan cloud.

\section{Conclusions and discussion}

In this paper we were able to construct a complete linear secular theory for Trojan-type motion (i.e. based on expansions of the disturbing potential truncated at degree two in eccentricities and inclinations). This was achieved through the use of a Hamiltonian formulation which allowed us to generalise the theory presented in Paper I by including, in a rigorous way, the effect of an oblate central mass and the secular perturbations from additional massive bodies.

Using our theory we were able to locate secular resonances inside the co-orbital regions of the uranian satellites and the planets Mercury to Neptune. Comparison with numerical integrations showed that these locations are reasonably accurate and that secular resonances seem to play a major role in determining the stability of Trojan orbits. 
We end by remarking that a next necessary step would be to develop a non-linear secular theory. In particular this would allow us to obtain even more accurate locations for these secular resonances (i.e. as functions of not only the proper semi-major axis, but also the proper eccentricity and the proper inclination). Moreover, a non-linear secular theory is also essential for application to the co-orbital structures with high eccentricity and/or inclination, studied by Namouni (1999).

Acknowledgements. I thank A. Morbidelli, D. Nesvorny and the reviewer, D. Hamilton, for useful comments. This work was supported by grants PRAXIS XXI/BD/5072/95 and SFRH/BPD/1586/2000 from the Fundação para a Ciência e a Tecnologia, Portugal.

\section{References}

Brankin, R. W., Dormand, J. R., Gladwell, I., Prince, P. J., \& Seward, W. L. 1987, NAG Technical Report, TR13/87

Brouwer, D., \& Clemence, G. M. 1961, Methods of Celestial Mechanics (Academic Press, New York)
Brouwer, D., \& Van Woerkom, A. J. J. 1950, Astron. Papers Amer. Ephem., XIII, 81

Dermott, S. F., \& Murray, C. D. 1981, Icarus, 48, 1

Gladman, B. 1993, Icarus, 106, 247

Greenberg, R. 1981, AJ, 86, 912

Hénon, M., \& Petit, J. M. 1986, Celest. Mech., 38, 67

Kozai, Y. 1959, AJ, 64, 367

Lagrange, J. L. 1867-1892 (Ouvres, Gauthier-Villars, Paris)

Laskar, J. 1988, A\&A, 198, 341

Lichtenberg, A. J., \& Lieberman, M. A. 1983, Regular and Stochastic Motion (Springer-Verlag, New York)

Malhotra, R., Fox, K., Murray, C. D., \& Nicholson, P. D. 1989, A\&A, 221, 348

Marzari, F., \& Scholl, H. 2000, Icarus, 146, 232

Michel, P. 1997, A\&A, 325, L5

Milani, A. 1994, The dynamics of the Trojan asteroids, in Asteroids Comets Meteors 1993, ed. A. Milani, et al., 159

Morais, M. H. M. 1999, A\&A, 350, 318

Namouni, F. 1999, Icarus, 137, 293

Yoder, C. F. 1979, Icarus, 40, 341

Yoder, C. F., Colombo, G., Synnott, S. P., \& Yoder, K. A. 1983, Icarus, 53, 431 